\title{
Morfología de la primera zoea de los cangrejos marinos Petrolisthes haigae y P. nobilii (Decapoda: Porcellanidae)
}

\author{
Gonzalo Hernández*, Juan Bolaños, Isabel Magán \& Karen Graterol \\ Escuela de Ciencias Aplicadas del Mar, Universidad de Oriente, Nueva Esparta, Apartado postal 074, La Asunción \\ 6311, Isla Margarita, Venezuela; gonzalo@c-com.net.ve; politus@cantv.net
}

Recibido 21-XI-2006. Corregido 23-III-2007. Aceptado 14-V-2007.

\begin{abstract}
Morphology of the first zoea of the marine crabs Petrolisthes haigae and $P$. nobilii (Decapoda: Porcellanidae). The porcellanid crabs Petrolisthes haigae Chace 1962, and P. nobilii Haig 1960, are shallowwater species distributed along the tropical Eastern Pacific shores. Larvae of both species from eight and four females, collected at Taboga and Naos Islands, respectively, were hatched in the laboratory. First zoeal stages of these species are described and illustrated for the first time and compared to previously first zoeae of other congeneric species from western Panama. Characters of the first zoeae of both species most closely agree with those Osawa's (2002) Group 4 of larvae. Rev. Biol. Trop. 55 (3-4): 879-887. Epub 2007 December, 28.
\end{abstract}

Key words: larvae, Porcellanidae, Petrolisthes, zoea, Eastern Pacific.

Petrolisthes es el género más diversificado de la familia Porcellanidae con más de un centenar de especies válidas. Este género está bien representado en el Pacífico tropical oriental, con unas 30 especies, de las cuales 19 se han reportado para las costas occidentales de Panamá (Hendrickx y Harvey 1999).

La morfología de las zoeas del género Petrolisthes es la mejor conocida de los Porcellanidae, con documentación para 32 especies. Entre éstas, seis han sido objeto de hallazgos en el Pacífico panameño: P. tridentatus Stimpson 1858, P. armatus (Gibbes 1850), P. platymerus Haig 1960, P. zacae Haig 1968, P. tonsorius Haig 1960 y P. robsonae Glassell 1945.

Petrolisthes haigae Chace 1962, ha sido reportada desde la Isla de San Francisquito y Guaymas (Sonora, Golfo de California, México), hasta Bahía Santa Elena (Ecuador), e Islas Revillagigedo, Clipperton, Malpelo y Galápagos, mientras que $P$. nobilii Haig 1960 ha sido objeto de reportes desde Cabeza Ballena
(Baja California Sur, Golfo de California, México), hasta Bahía Santa Elena (Ecuador) e Isla Isabel (Hendrickx y Harvey 1999).

En esta investigación se describe e ilustra la morfología de la primera zoea de $P$. haigae y de $P$. nobilii, y se les compara con el estadio equivalente de otras especies congenéricas del occidente panameño, con el propósito de facilitar su identificación en las muestras de plancton de esta zona. También se discute la afinidad de estas dos especies dentro de los grupos larvarios de Petrolisthes.

\section{MATERIALES Y MÉTODOS}

Ocho hembras ovígeras de $P$. haigae y cuatro de $P$. nobilii fueron recolectadas como simbiontes del coral Pocillopora damicornis en Isla Taboga y en el intermareal rocoso de Isla Naos (Panamá), respectivamente. Estas hembras fueron mantenidas individualmente en vasos con $500 \mathrm{ml}$ de agua de mar. Después de la 
eclosión las larvas fueron colocadas en grupos de 10 en recipientes con $250 \mathrm{ml}$ de agua de mar, a temperatura ambiente. El agua fue renovada diariamente y las larvas fueron alimentadas con nauplios recién nacidos de Artemia. Debido a la deficiente calidad del agua de cultivo ninguna de las larvas sobrevivió más de cuatro días ni mudó al siguiente estadio (el Instituto Smithsoniano de Investigación Tropical, en Panamá, se encontraba en operaciones de mantenimiento en ese entonces). Las larvas fueron preservadas en formalina al $4 \%$ y fueron medidas y analizadas en un microscopio compuesto Olympus BM-50 provisto de una camera lucida. Las hembras ovígeras y muestras de su progenie fueron depositadas en el Laboratorio de Crustáceos (Universidad de Oriente, Núcleo Nueva Esparta Venezuela) e identificadas con los códigos LP101(P. haigae) y LP-102 (P. nobilii).

La terminología para los diferentes tipos de setas y la metodología utilizada para medir el largo del caparazón (LC), de la espina rostral (ER) y de las espinas posteriores (EP) son las mismas expresadas por Hernández et al. (1998, 2000). Se estimó la media aritmética de los especímenes medidos. Las fórmulas setales fueron expresadas siguiendo un ordenamiento proximal-distal en cada artejo. Las setas dorsales del endopodito de los maxilípedos 1 y 2, así como las setas apicales del escafognatito de la maxila fueron representadas con números romanos.

\section{RESULTADOS}

\section{Petrolisthes haigae}

\section{Chace 1962 (Figs. 1,2)}

Primera zoea: Tamaño $\mathrm{LC}=1.50 \mathrm{~mm}$; $\mathrm{ER}=5.41 \mathrm{~mm} ; \mathrm{EP}=1.62 \mathrm{~mm}$. Especímenes medidos: 15. Especímenes examinados: 10.

Caparazón (Figs. 1A-D). Con dos pares de setas en el dorso; márgenes ventrales inermes. Espina rostral 3.2 a 3.6 veces el largo del caparazón, con numerosas espínulas cortas en toda su extensión. Espinas posteriores tan largas como el caparazón, con 12 a 14 espínulas en su margen ventral. Ojos sésiles.
Anténula (Fig. 2A). Unirrámea, alargada, con tres estetascos subiguales, dos setas setulosas y una seta simple más corta en el extremo distal.

Antena (Fig. 2B). Birrámea. Endopodito fusionado al protopodito, con una seta simple subterminal. Exopodito 1.4 veces más largo que el endopodito, provisto de dos setas setulosas y una espina subterminal.

Mandíbulas (Fig. 2C). Asimétricamente dentadas. Procesos incisivo y molar bien desarrollados. Palpo ausente.

Maxílula (Fig. 2D). Endito coxal con ocho setas (seis setulodenticuladas, dos setulosas). Endito basial con nueve setas (seis denticuladas, tres setulosas). Endopodito insegmentado, con 1+2 setas setulosas; espina mesial presente. Exopodito ausente.

Maxila (Fig. 2E). Enditos coxal y basial bilobulados, con 7,4 y 7,7 setas setulosas en los lóbulos proximal y distal, respectivamente. Endopodito insegmentado, con $3+2+4$ setas setulosas. Escafognatito con $6+\mathrm{I}$ setas setulosas. Microtrichia en ambas ramas y enditos como se ilustra.

Maxilípedo 1 (Fig. 2F). Birrámeo. Coxopodito inerme. Basipodito con $1+1+2+3$ setas setulosas. Endopodito 4-segmentado con 3,3,2+4,7+I setas setulosas. Exopodito 2-segmentado, con cuatro setas setulosas ubicadas terminalmente.

Maxilípedo 2 (Fig. 2G). Birrámeo. Coxopodito inerme. Basipodito con $1+1$ setas setulosas. Endopodito 4-segmentado con 1,2,1+2,5+I setas setulosas. Exopodito 2-segmentado, con cuatro setas setulosas ubicadas terminalmente.

Maxilípedo 3 y pereiópodos. Presentes como rudimentos indiferenciados.

Abdomen (Fig. 1E). Compuesto por cinco somitos, los dos últimos con espinas posterolaterales. Márgenes posterodorsales enteros y sin setas. Pleópodos ausentes.

Telson (Figs. 1F-L). Tan largo como ancho. Seta anomúrica presente. Espinas laterales bífidas (Fig. 1G). Cinco pares de setas mayores setuloaserradas (Figs. 1H-L); prominencia central con el quinto par de setas mayores y 


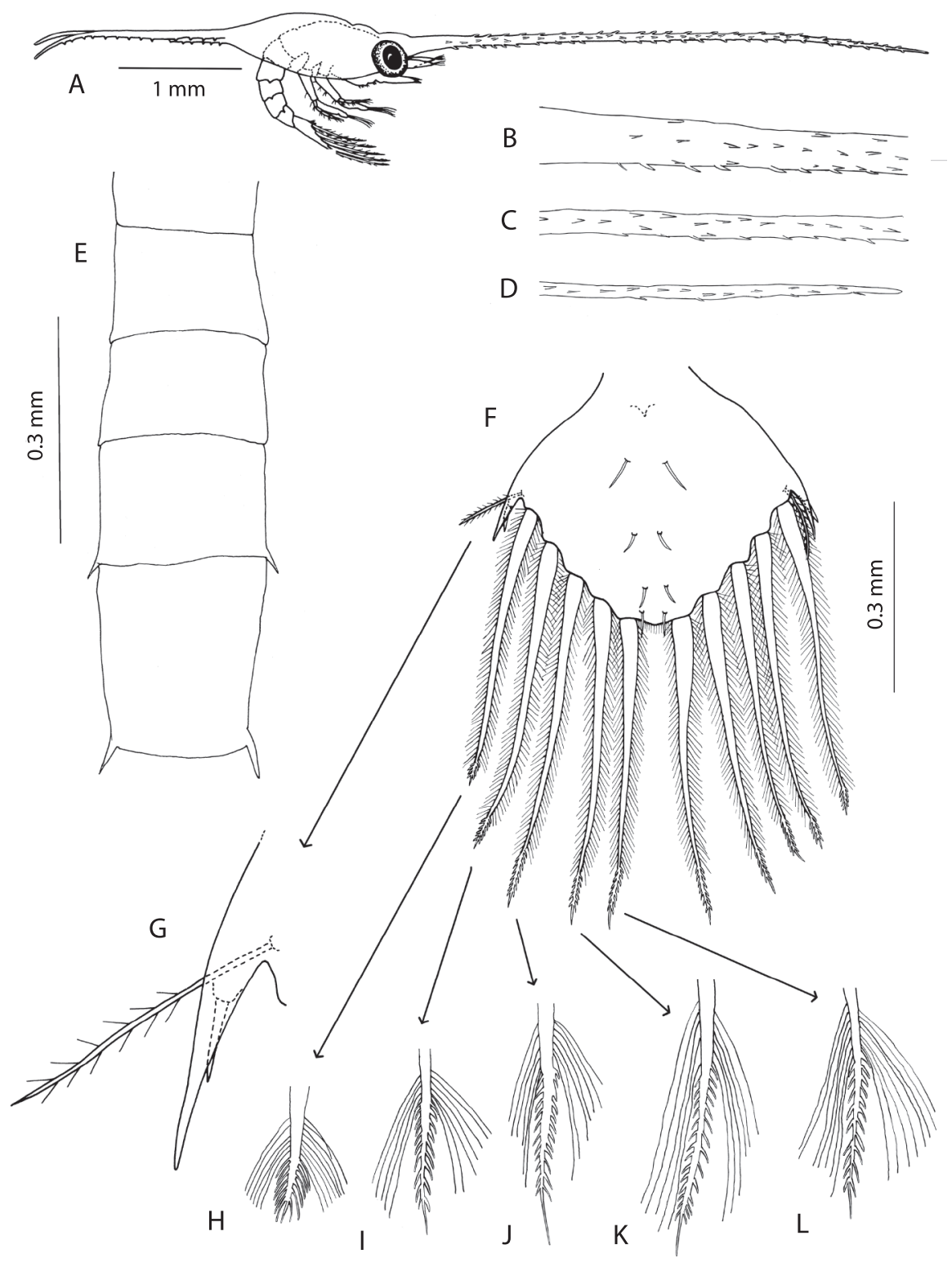

Fig. 1. Petrolisthes haigae. Primera zoea: vista lateral de un ejemplar (A); porciones proximal (B), mesial (C) y distal (D) del rostro; vista dorsal del abdomen (E) y del telson (F); detalle de la espina lateral del telson (G) y ápice de las setas mayores del telson (H-L). 

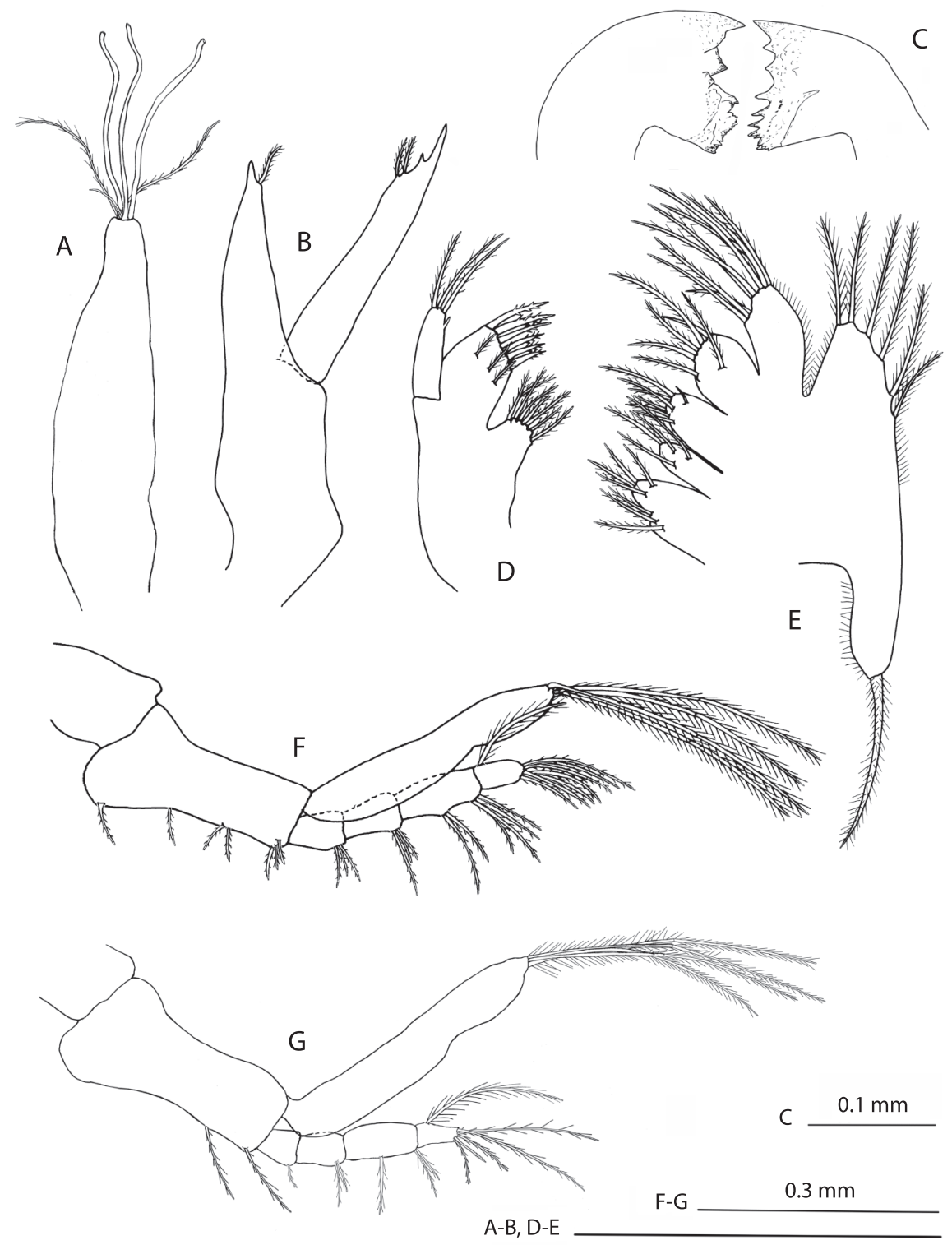

Fig. 2. Petrolisthes haigae. Primera zoea: anténula (A), antena (B), mandíbulas (C), maxílula (D), maxila (E), maxilípedo 1 (F) y maxilípedo 2 (G).

microtrichia. Cuatro pares de setas dorsales y espina anal presentes.

\section{Petrolisthes nobilii \\ Haig 1960 (Figs. 3,4)}

Primer estadio de zoea: Tamaño $\mathrm{LC}=$ $1.06 \mathrm{~mm} ; \mathrm{ER}=3.35 \mathrm{~mm} ; \mathrm{EP}=1.38 \mathrm{~mm}$.
Especímenes medidos: 10. Especímenes examinados: 6.

Caparazón (Figs. 3A-D). Con dos pares de setas en el dorso; márgenes ventrales inermes. Espina rostral 2.8 a 3.6 veces más larga que el caparazón, con numerosas espínulas en toda su extensión, excepto en el ápice. Espinas posteriores 1.2 a 1.4 veces más largas que el 

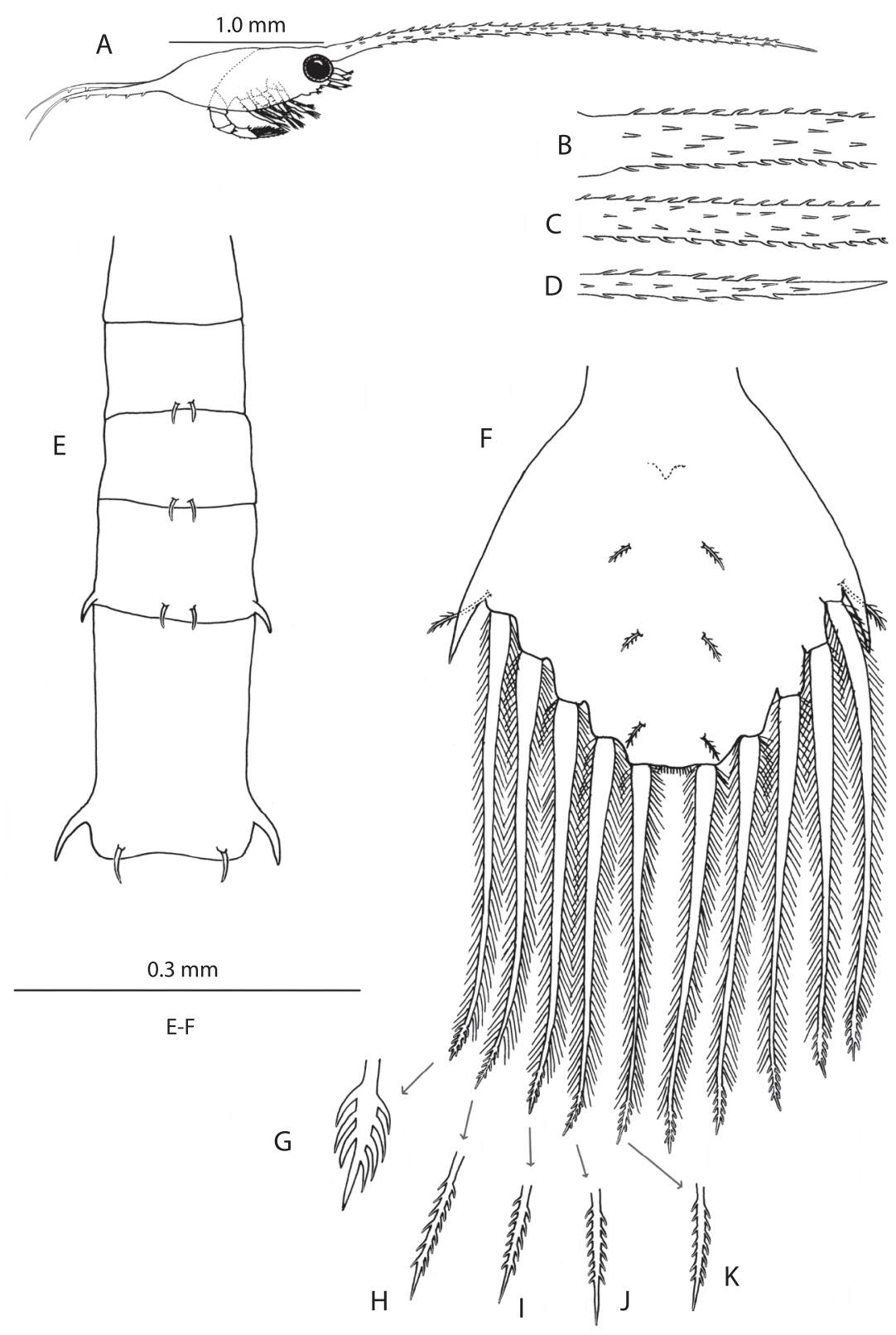

Fig. 3. Petrolisthes nobilii. Primera zoea : vista lateral de un ejemplar (A); porciones proximal (B), mesial (C) y distal (D) del rostro; vista dorsal del abdomen (E) y del telson $(\mathrm{F})$; detalle del ápice de las setas mayores del telson (G-K).

caparazón, con cuatro a siete espínulas ventralmente. Ojos sésiles.

Anténula (Fig. 4A). Unirrámea, alargada, con tres estetascos subiguales, dos setas setulosas y una seta simple más pequeña, en el extremo distal.
Antena (Fig. 4B). Birrámea. Endopodito fusionado al protopodito, con una seta simple en posición subterminal. Exopodito 1.4 veces más largo que el endopodito, provisto de dos setas setulosas. 


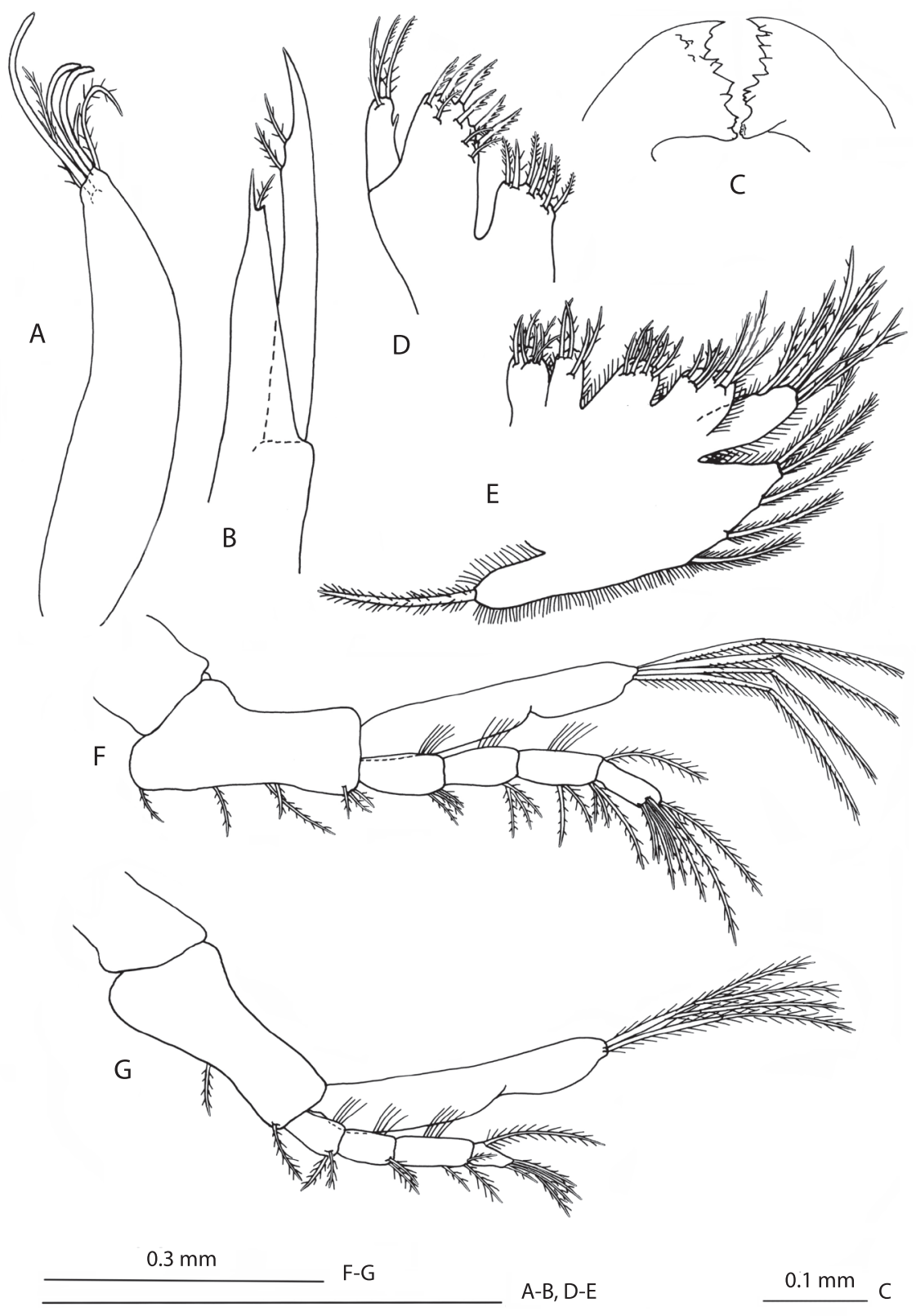

Fig. 4. Petrolisthes nobilii. Primera zoea: anténula (A), antena (B), mandíbulas (C), maxílula (D), maxila (E), maxilípedo 1 (F) y maxilípedo 2 (G).

Mandíbulas (Fig. 4C). Asimétricamente dentadas. Procesos incisivo y molar bien desarrollado. Palpo ausente.

Maxílula (Fig. 4D). Endito coxal con ocho setas (seis setulodenticuladas, dos setulosas). Endito basial con nueve setas (seis denticuladas, tres setulosas). Endopodito insegmentado, con tres setas setulosas y espina mesial presente. Exopodito ausente.

Maxila (Fig. 4E). Enditos coxal y basial bilobulados, con 7,4 y 7,7 setas setulosas en los lóbulos proximal y distal, respectivamente. 
Endopodito insegmentado, con $3+3+3$ setas setulosas. Escafognatito con $5+\mathrm{I}$ setas setulosas,

Maxilípedo 1 (Fig. 4F). Birrámeo. Coxopodito inerme. Basipodito con $1+1+2+3$ setas setulosas en posición ventral. Endopodito 4-segmentado con 3,3,2+3,7+I setas setulosas. Exopodito 2-segmentado, con cuatro setas setulosas ubicadas terminalmente.

Maxilípedo 2 (Fig. 4G). Birrámeo. Coxopodito inerme. Basipodito con $1+1$ setas setulosas. Endopodito 4-segmentado con 2,2,1+2,5+1 setas setulosas. Exopodito 2-segmentado, con cuatro setas setulosas ubicadas terminalmente.

Maxilípedo 3 y pereiópodos. Presentes como rudimentos indiferenciados.

Abdomen (Fig. 3E). Compuesto por cinco somitos, los dos últimos con espinas porterolaterales. Márgenes posterodorsales enteros. Un par de setas posterodorsalmente en los somitos 2 a 5. Pleópodos ausentes.

Telson (Figs. 3F-K). Tan largo como ancho. Seta anomúrica presente. Espinas laterales simples. Cinco pares de setas mayores setuloaserradas (Figs. 3O-S); prominencia central con el quinto par de setas mayores y microtrichia. Tres pares de setas dorsales y espina anal presentes.

\section{DISCUSIÓN}

Osawa (1995) estudió la morfología larvaria de 19 especies de Petrolisthes y las dividió en seis grupos con base en seis estructuras morfológicas del primer y segundo estadios de zoea. Este esquema ha sido modificado por Osawa (1997) al incluir otras cuatro especies, y por Fujita et al. (2002) al incorporar otras especies de este género hasta completar 28, así como al designar un nuevo grupo para $P$. unilobatus Henderson 1888.

De los grupos propuestos por Fujita et al. (2002), el Grupo 4 es el que contiene mayor número de especies. Este grupo se ha visto incrementado con la incorporación de $P$. politus (Gray 1831) (Hernández et al. 2000), P. caribensis Werding 1983 (Kraus et al. 2004) y P. robsonae Glassell 1945 (García-Guerrero et al. 2005). Las 24 especies que ahora conforman el Grupo 4 comparten las siguientes características: primera zoea con (1) escafognatito de la maxila con sólo una seta en el lóbulo proximal, (2) primer par de maxilípedos con endopodito 4-segmentado, (3) solamente el cuarto segmento del primer par de maxilípedos con seta dorsal, (4) todas las setas mayores del telson son setuloaserradas, y la segunda zoea con (5) mandíbulas provistas de palpo, y (6) una seta adicional en el margen posterior del telson (fórmula setal: $5+1+5$ ).

El Grupo 5, compuesto únicamente por P. platymerus Haig 1960, de acuerdo con el desarrollo larvario descrito por Gore (1972b), difiere del Grupo 4 tan sólo por la constitución del telson en la segunda zoea, ya que en $P$. platymerus no hay seta adicional en el margen posterior del telson (fórmula setal: $5+0+5$ ) (Osawa 1995, Fujita et al. 2002). Además, $P$. platymerus presenta ápices romos, en vez de agudos, en el endopodito y exopodito de la antena de ambos estadios de zoea, lo cual no ha sido descrito para ninguna otra especie de Porcellanidae y esta característica bien podría ser utilizada para la tipificación de este grupo.

Con base a estas apreciaciones y a pesar de que la morfología del segundo estadio de zoea de $P$. haigae y $P$. nobilii permanece ignota, se podría esperar que estas dos especies formen parte del Grupo 4 (Osawa 1995, Fujita et al. 2002), elevándose el número de especies allí asignadas a 26 (equivalentes a $86 \%$ de un total de 33 especies). La descripción de la morfología de la segunda zoea de estas dos especies será la herramienta que permita darle forma definitiva a esta propuesta.

La primera zoea de $P$. haigae puede ser diferenciada de su equivalente en $P$. tridentatus (ver Gore 1971), P. armatus (ver Gore 1972a), $P$. platymerus (ver Gore 1972b), P. zacae (ver Gore 1975), P. tonsorius (ver Pellegrini y Gamba 1985) y $P$. robsonae (ver García-Guerrero et al. 2005), hasta ahora las únicas especies congenéricas presentes en la zona del Pacífico panameño con morfología larvaria documentada, por la combinación de un conjunto de características, pero especialmente porque la espina lateral 
de su telson es bífida, en vez de simple. Se trata de la primera especie de Porcellanidae del Pacífico oriental hallada con espina lateral bífida en el telson de la primera zoea. Este tipo de configuración sólo había sido reseñado en las siguientes especies del Indo-Pacífico: $P$. rufescens (Heller 1861) (ver Yaqoob 1974), $P$. lamarckii (Leach 1820) (ver Yaqoob 1979), $P$. coccineus (Owen 1839) (ver Osawa 1995), $P$. melini Miyake y Nakasone, 1966 y P. pubescens Stimpson, 1858 (ver Osawa 1995), y P. asiaticus (Leach 1820), P. hastatus Stimpson, 1858, P. moluccensis (De Man 1888) y P. tomentosus (Dana 1852) (ver Osawa 1997), y en las siguientes especies del Mar Caribe: P. politus (Gray 1831) (ver Hernández et al. 2000), P. galathinus (Bosc 1802) (ver Muñoz 2001) y P. caribensis Werding, 1983 (ver Kraus et al. 2004).

En cambio, para diferenciar la primera zoea de $P$. nobilii de su equivalente en las especies congenéricas antes mencionadas del Pacífico panameño con morfología larvaria documentada, se requiere comparar y combinar varias propiedades estructurales. Por ejemplo, difiere de $P$. tonsorius y $P$. tridentatus por la ausencia del proceso ganchoso en el coxopodito del primer par de maxilípedos; se diferencia de $P$. platymerus por la ausencia de endopodito y exopodito antenales romos y por la ausencia de setas en el coxopodito del maxilípedo 1; se puede distinguir de $P$. robsonae por el número de estetascos y la fórmula setal de los enditos maxilares y basipodito del maxilípedo 2; de $P$. zacae se diferencia por la ausencia de espinas en el exopodito y endopodito de la antena y el número de setas en el endito coxal de la maxílula, difiere de $P$. armatus en que las espinas posteriores del caparazón son más largas que el caparazón propiamente dicho (LC), por la presencia de tres setas en la anténula (en lugar de dos) y de ocho setas en el endito coxal de la maxílula (en lugar de siete); mientras que de $P$. haigae, como ya se señaló, se distingue porque su telson tiene espinas laterales simples en vez de bífidas.

\section{AGRADECIMIENTOS}

Esta investigación fue subvencionada por la Universidad de Oriente (Venezuela). Facilidades de campo y laboratorio fueron gentilmente concedidas por Juan A. Gómez y Hanzel Villalaz (Centro de Biología Marina y Limnología, Universidad de Panamá), y el Instituto Smithsoniano de Investigación Tropical (Panamá). Ana Raquel Muñoz contribuyó con la elaboración de algunas ilustraciones. La lectura crítica que hicieron los revisores sirvió para corregir detalles de importancia y enriquecer el texto.

\section{RESUMEN}

Los "cangrejos porcelana" Petrolisthes haigae Chace 1962 y P. nobilii Haig 1960, son especies de aguas poco profundas distribuidas a lo largo de las costas del Pacífico Este Tropical. Recolectamos larvas de ambas especies, de ocho y cuatro hembras, en las islas de Taboga y Naos, respectivamente. Las criamos en el laboratorio para describir e ilustrar los primeros estados de zoea de estas especies fueron, hasta ahora desconocidos. Las comparamos con los primeros estadios de zoea de otras especies del género en el oeste de Panamá y concluimos que las características de la primera zoea de ambas especies se asemejan a las del grupo 4 de larvas propuesto por Osawa en 2002.

Palabras clave: larva, Porcellanidae, Petrolisthes, zoea, Pacífico oriental.

\section{REFERENCIAS}

Fujita, Y., S. Shokita \& M. Osawa. 2002. Complete larval development of Petrolisthes unilobatus reared under laboratory conditions (Decapoda: Anomura: Porcellanidae). J. Crust. Biol. 22: 567-580.

García-Guerrero, M.U., J.A. Cuesta, M.E. Hendrickx \& A. Rodríguez. 2005. Larval development of the eastern Pacific anomuran crab Petrolisthes robsonae (Crustacea: Decapoda: Anomura: Porcellanidae) described from laboratory reared material. J. Mar. Biol. Ass. U.K. 85: 339-349. 
Gore, R.H. 1971. Petrolisthes tridentatus: The development of larvae from a Pacific specimen in laboratory culture with a discussion of larval characters in the genus (Crustacea: Decapoda: Porcellanidae). Biol. Bull. 141: 485-501.

Gore, R.H. 1972a. Petrolisthes armatus (Gibbes 1850): The development under laboratory conditions of larvae from a Pacific specimen (Decapoda, Porcellanidae). Crustaceana 22: 67-83.

Gore, R.H. 1972b. Petrolisthes platymerus: The development of larvae in laboratory culture (Crustacea: Decapoda: Porcellanidae). Bull. Mar. Sci. 22: 336-354.

Gore, R.H. 1975. Petrolisthes zacae Haig, 1968 (Crustacea, Decapoda, Porcellanidae): the development of larvae in the laboratory. Pac. Sci. 29: 181-196.

Hendrickx, M.E. \& A.W. Harvey. 1999. Checklist of anomuran crabs (Crustacea: Decapoda) from the eastern tropical Pacific. Belg. J. Zool. 129: 363-389.

Hernández, G., J. Bolaños, K. Graterol \& C. Lira. 2000. The larval development of Petrolisthes politus (Gray 1831) (Crustacea: Decapoda: Porcellanidae) under laboratory conditions. Stud. Neotrop. Fauna Environ. 35: 143-155.

Hernández, G., K. Graterol, A. Álvarez \& J. Bolaños. 1998. Larval development of Porcellana sayana (Leach 1820) (Crustacea: Decapoda: Porcellanidae) under laboratory conditions. Nauplius 6: 101-118.
Kraus, H., A. Hiller, \& N. Cruz. 2004. The zoeal stages of Petrolisthes caribensis Werding, 1983 reared under laboratory conditions (Decapoda: Anomura: Porcellanidae). Stud. Neotrop. Fauna Environ. 39: 85-90.

Muñoz, A.R. 2001. Desarrollo larvario de Petrolisthes galathinus (Bosc 1802) (Decapoda: Anomura: Porcellanidae) en condiciones de laboratorio. Tesis Lic. Biología, Universidad de Oriente, Cumaná, Venezuela.

Osawa, M. 1995. Larval development of four Petrolisthes species (Decapoda: Anomura: Porcellanidae) under laboratory conditions, with comments on the larvae of the genus. Crust. Res. 24: 157-187.

Osawa, M. 1997. Zoeal development of four Indo-West Pacific species of Petrolisthes (Crustacea: Decapoda: Porcellanidae). Spec. Div. 2: 121-143.

Pellegrini, N.C. \& A.L. Gamba. 1985. Larval development of Petrolisthes tonsorius Haig 1960, under laboratory conditions (Decapoda, Porcellanidae). Crustaceana 49: 251-267.

Yaqoob, M. 1974. Larval development of Petrolisthes rufescens (Heller 1861) (Decapoda: Porcellanidae) under laboratory conditions. Pakistan J. Zool. 6: 47-61.

Yaqoob, M. 1979. Rearing of Petrolisthes lamarcki (Leach 1820) under laboratory conditions (Decapoda: Porcellanidae). Crustaceana 37: 253-264. 
